\title{
A Multiple Loop Feedback Gm-C Bandpass Filter for Wavelet Transform Implementation
}

\author{
Wenshan Zhao ${ }^{1,2}$, Yichuang Sun ${ }^{1}, \mathrm{Xi} \mathrm{Zhu}^{1}$, and Yigang $\mathrm{He}^{2}$ \\ 1. School of ECEE, University of Hertfordshire, Hatfield, Herts AL10 9AB, UK \\ 2. College of Electrical \& Information Engineering, Hunan University, Changsha, Hunan 410082, China \\ Email: shmilyzhyao@yahoo.com.cn, Y.Sun@herts.ac.uk
}

\begin{abstract}
A general method for analogue implementation of high-frequency wavelet transform is proposed in which the wavelet transform is synthesized by a bank of $\mathrm{Gm}-\mathrm{C}$ bandpass filters whose impulse responses are the mother wavelet and its dilations. The proposed approach employs the inverse follow-the-leader-feedback multiple loop feedback structure for filter design to lower the magnitude sensitivity and facilitate the arbitrary rational function realization. The Marr wavelet is used as an example in this paper to illustrate design details. The wavelet filter is simulated using TSMC $1.8 \mathrm{~V} 0.18 \mu \mathrm{m}$ CMOS technology. Simulation results show performance values obtained.
\end{abstract}

\section{INTRODUCTION}

Wavelet transform (WT), developed in the late of 1980s, has been demonstrated to be a high performance signal processing technique and an effective tool in various fields [1]. Over the past few years, applications of WT in high-frequency signal processing, in particular, have attracted much attention [2-6]. However, a principal obstacle to the wider utilization of these algorithms is the heavy computational burden, which limits speed and real-time signal processing. In recent years, hardware implementations have been exploited, mostly concentrating on digital circuit implementations [4-6]. Although successful in many aspects, these designs suffer from the effect of large power dissipation associated with the required analogue-digital converter.

To alleviate the difficulties associated with such digital designs, this paper proposes a method that employs a bank of continuous-time $\mathrm{Gm}-\mathrm{C}$ bandpass filters whose impulse responses are the wavelet bases (i.e. wavelet filter). $\mathrm{Gm}-\mathrm{C}$ filters are well suited for high-frequency operation with relatively low power consumption and are widely regarded as the optimum technique for CMOS integrated high-frequency continuous-time filters [7]. Based on the optimal approximation method proposed in [8], this paper synthesizes the wavelet base with stable and realizable rational functions, and then implements it using a fully-differential Gm-C bandpass filter based on the inverse follow-the-leaderfeedback (IFLF) multiple loop feedback (MLF) structure.

The wavelet design procedure is discussed in Section 2. Section 3 focuses on the wavelet base approximation, where the Marr wavelet (i.e. Mexican hat wavelet) is selected as an example to elaborate the approximation procedure. Next, Section 4 describes the complete wavelet filter design using Gm-C technique, in which a fully-differential linear transconductor is employed as the $\mathrm{Gm}$ cell in order to minimize the nonlinearity and reject even-order distortion. The simulation results are given in Section 5. Finally, Section 6 presents the conclusions.

\section{PRINCIPLE OF OPERATION FOR WAVELET TRANSFORM IMPLEMENTATION}

Assuming $\psi(\mathrm{t})$ is the mother wavelet (or wavelet base), the wavelet transform of the signal $\mathrm{x}(\mathrm{t})$ at the scale $a$ and timeshift $b$ can be defined by convolving $\mathrm{x}(\mathrm{t})$ with a dilated wavelet [1],

$$
W T_{x}(a, b)=a^{-1} \int x(t) \psi\left(\frac{b-t}{a}\right) d t
$$

The coefficient, $1 / a$, maintains the amplitude of frequency response of $\psi_{a, b}=a^{-1} \psi\left(\frac{b-t}{a}\right)$ across different scales. Equivalently, (1) can be expressed in terms of the Fourier transforms of the signal and wavelet

$$
\mathrm{WT}_{\mathrm{x}}(a, \tau)=[1 /(2 \pi)] \int X(\omega) \Psi^{*}(a \omega) \exp (j \omega \tau) d \omega
$$

Due to the characteristic of satisfying the admissibility criteria [1], the wavelets are inherently bandpass filters in the Fourier domain, defined as wavelet filters. Hence, the WT can be carried out simply by using a bank of bandpass filters whose impulse responses are the mother wavelet and its dilated versions. As an example, the Marr WT system is presented in this paper. 


\section{APPROXIMATION OF WAVELET BASES}

The Marr wavelet at scale $a$ in time-domain and frequencydomain can be defined respectively as [1]

$$
\begin{aligned}
& \psi_{a}(t)=\frac{1}{a} \psi\left(\frac{t}{a}\right)=\frac{1}{a} \frac{2}{\pi^{1 / 4} \sqrt{3}}\left[1-\left(\frac{t}{a}\right)^{2}\right] \exp \left(-\frac{t^{2}}{2 a^{2}}\right) \\
& \Psi_{a}(\omega)=\Psi(a \omega)=\pi^{\frac{1}{4}} \sqrt{\frac{8}{3}} a^{2} \omega^{2} \exp \left(-a^{2} \omega^{2} / 2\right)
\end{aligned}
$$

It is obvious that the Marr wavelet $\psi(t)$ is non-causal because of its symmetry around $t=0$, which means incapability of being implemented by analogue circuits. For the purpose of making wavelet causal, a time delay $T$ should be introduced to shift $\psi(t)$ to the right. It is worth noting that this operation will delay the filter output, without change in amplitude.

Denoting $s=j \omega$ and $T=t_{0}$, the desired transfer function derived from (4) can be obtained as

$$
H(s)=\frac{-\pi^{\frac{1}{4}} \sqrt{8 / 3} a^{2} s^{2}}{e^{s t_{0}-a^{2} s^{2} / 2}}
$$

Using Maclaurin series expansion for the denominator of the expression in (5), a stable and realizable approximation of this transfer function which can be implemented by means of standard filters is given as

$$
H(s)=\frac{-\pi^{\frac{1}{4}} \sqrt{8 / 3} a^{2} s^{2}}{1+s t_{0}+\left(\frac{t_{0}^{2}}{2}-\frac{a^{2}}{2}\right) s^{2}+\left(\frac{t_{0}^{3}}{6}-\frac{t_{0} a^{2}}{2}\right) s^{3}+\cdots}
$$

The factors in (6) are partly inter-related in which suitable $t_{0}$ values depend strongly upon the filter order [8]. For the case of $a=1$, the value $t_{0}=4$ is found to give a stable transfer function for up to a $7^{\text {th }}$ order approximation, which can be expressed as

$$
H(s)=\frac{-2.18 s^{2}}{0.234 s^{7}+1.34 s^{6}+3.7 s^{5}+6.79 s^{4}+8.67 s^{3}+7.5 s^{2}+4 s+1}
$$

\section{DESIGN OF WAVELET BANDPASS IFLF GM-C FILTERS}

\section{A. Fully-Differential Linear Transconductor}

To optimize the linearity and boost up DC gain, the fullydifferential transconductor [9] with active biasing and a negative compensation load $\left(\mathrm{G}_{\mathrm{L}}\right)$ implemented using the same transconductor is employed as the $\mathrm{Gm}$ cell of the bandpass filter, as shown in Fig. 1.

In the $\mathrm{Gm}$ cell, $\mathrm{M}_{1}$ and $\mathrm{M}_{2}$ are the input differential pair transistors. Transistors $\mathrm{M}_{3}-\mathrm{M}_{6}$ act as the active biasing circuitry in such a way that the total bias current drawn by $\mathrm{M}_{3}-\mathrm{M}_{6}$ is less for larger signals. They share the same bias current source with the differential pair $\mathrm{M}_{1}-\mathrm{M}_{2}$. When the signal amplitude is small, $\mathrm{M}_{5}$ and $\mathrm{M}_{6}$ are in triode region while $\mathrm{M}_{3}$ and $\mathrm{M}_{4}$ are in saturation. The $\mathrm{M}_{3}-\mathrm{M}_{6}$ branches take away some of the bias current. When the signal amplitude is positive and large, $\mathrm{M}_{5}$ will be cut off and $\mathrm{M}_{6}$ will become saturated. A smaller proportion of the total bias current will be drawn by $\mathrm{M}_{3}-\mathrm{M}_{6}$. This leads to larger currents in $\mathrm{M}_{1}$ and $\mathrm{M}_{2}$ to compensate for the drop of the transconductance. The sizing of the transistors must be optimized for maximum linearity [9].

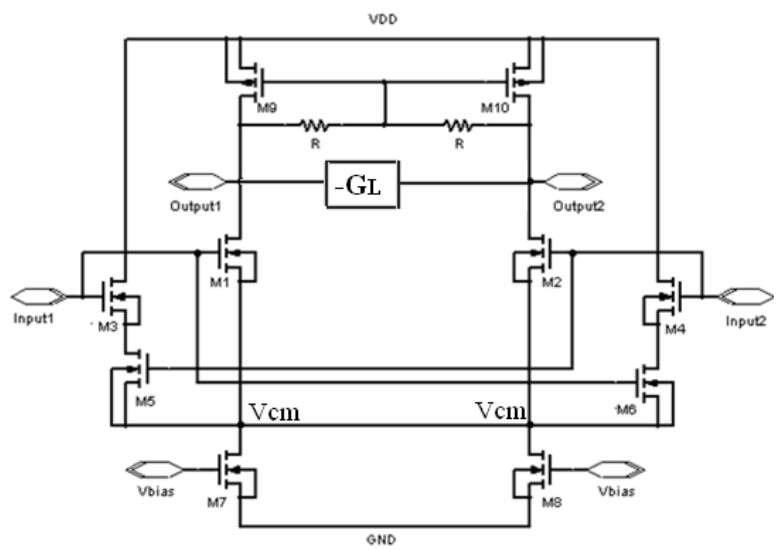

Figure 1. Fully-differential transconductor

Let $\mathrm{K}_{3,4}$ be $\mathrm{C}$ times of $\mathrm{K}_{5}, 6$ for higher linearity, where $K_{x}=\mu C_{o x} W_{x} / L_{x}$. Based on the analysis above, $\mathrm{I}_{\mathrm{D} 3}$ and $\mathrm{I}_{\mathrm{D} 5}$ have the following relationship

$$
\begin{aligned}
I_{D 3} & =I_{D 5} \Rightarrow 0.5 K_{3}\left(0.5 V_{i n}-V_{d 5}-V_{t}\right)^{2} \\
& =K_{5}\left[-\left(0.5 V_{i n}+V_{c m}+V_{t}\right) V_{d s 5}-0.5 V_{d s 5}^{2}\right]
\end{aligned}
$$

Let $\mathrm{V}_{\mathrm{cm}}+\mathrm{V}_{\mathrm{t}}=\mathrm{V}_{\mathrm{cm}}{ }^{*} \cdot \mathrm{I}_{\mathrm{D} 3}$ can be written as

$$
\begin{aligned}
I_{D 3} & =K_{3}\left[0.5 V_{i n}-\left(V_{d s 5}+V_{c m}^{*}\right)\right]^{2} \\
& =\frac{K_{3}}{4(C+1)^{2}}\left[2 V_{i n}-\sqrt{(1-3 C) V_{i n}^{2}+4(C+1) V_{i n} V_{c m}^{*}+4(C+1) V_{c m}^{2 *}}\right]^{2}
\end{aligned}
$$

$I_{D 4}$ has a similar expression except with $V_{\text {in }}$ being replaced by $-V_{\text {in }}$. Therefore, the output current can be expressed as:

$$
I_{\text {out }}=K_{1,2} \sqrt{\frac{I_{B}-\left[0.5 K_{1,2}-\frac{K_{3,4}}{2(C+1)^{2}}(5-3 C-4 \sqrt{C+1})\right] V_{\text {in }}^{2}}{2\left[K_{1,2}+(C+1) K_{3,4}\right]}} V_{\text {in }}
$$

where $I_{B}$ is the bias current.

Using Taylor series expansion, (10) can be written as

$$
I_{\text {out }} \approx \sqrt{\frac{K_{1,2}^{2} I_{B}}{2\left[K_{1,2}+(C+1) K_{3,4}\right.}} V_{\text {in }}-\frac{K_{1,2}^{2}-\frac{K_{1,2} K_{3,4}}{(C+1)^{2}}(3 C+4 \sqrt{C+1}-5)}{12 \sqrt{2\left[K_{1,2}+(C+1) K_{3,4}\right] I_{B}}} V_{\text {in }}^{3}
$$

Note that the even-order harmonic distortions are cancelled completely due to the symmetry of fully differential Gm cell. The total harmonic distortion is mainly determined by the third harmonic distortion (HD3). As seen from (11), HD3 can be cancelled as long as the following relationship is met,

$$
K_{1,2}^{2}=\frac{K_{1,2} \cdot K_{3,4}}{(C+1)^{2}}(3 C+4 \sqrt{C+1}-5)
$$


The value of variable $\mathrm{C}$ is chosen to give an optimal value of linearity. Therefore, the transconductance of the $\mathrm{Gm}$ cell shown in Fig. 1 is derived as

$$
G_{m} \approx \sqrt{\frac{K_{1,2}^{2} I_{B}}{2\left[K_{1,2}+(C+1) K_{3,4}\right]}}
$$

The differential loading $\mathrm{R}$ is made with two polysilicon resistors for better linearity and more constant loading toward different signal swings. A negative $\mathrm{Gm}$ cell $\left(-\mathrm{G}_{\mathrm{L}}\right)$ is connected in parallel to the transconductor output to enhance the output impedance. The design of the $-\mathrm{G}_{\mathrm{L}}$ cell is similar to that of the Gm cell except that the outputs are cross coupled to the inputs.

\section{B. Design of Wavelet Filter Based on IFLF Gm-C Topology}

The filter's performances not only rely on the characteristics of transconductors, but also strongly on the filter structure. So far, many filter architectures such as cascade and LC ladder simulation have been utilized in filter designs for high-frequency operation. As is well known, these designs suffer from the effect of higher magnitude sensitivity (cascade) and incapability of realizing real zeros directly (LC ladder simulation). It has been demonstrated that MLF approach is one of the solutions to these problems [7]. The IFLF MLF configuration [10,11] is therefore employed for the filter design in this paper to lower the magnitude sensitivity and facilitate the arbitrary rational function realization.

The desired Marr transfer function at $a=1$ obtained in Section 3 has the form of

$$
H(s)=\frac{A_{2} s^{2}}{B_{7} s^{7}+\cdots+B_{2} s^{2}+B_{1} s+1}
$$

The fully-differential realization of (14) using IFLF MLF structure with input distribution transconductors producing zeros is shown in Fig. 2.

Denoting $\tau_{j}=C_{j} / g_{j}$ and $\beta_{j}=g_{a j} / g_{j}$, the overall transfer function of the filter can be derived as

$$
H(s)=\frac{V_{o u t}}{V_{\text {in }}}=\frac{N(s)}{D(s)}
$$

Where $N(s)=-\beta_{3} \tau_{1} \tau_{2} s^{2}$

$D(s)=\tau_{1} \tau_{2} \tau_{3} \tau_{4} \tau_{5} \tau_{6} \tau_{7} s^{7}+\tau_{1} \tau_{2} \tau_{3} \tau_{4} \tau_{5} \tau_{6} s^{6}+\tau_{1} \tau_{2} \tau_{3} \tau_{4} \tau_{5} s^{5}$

$+\tau_{1} \tau_{2} \tau_{3} \tau_{4} s^{4}+\tau_{1} \tau_{2} \tau_{3} s^{3}+\tau_{1} \tau_{2} s^{2}+\tau_{1} s+1$

Using the coefficient matching between (15) and the desired transfer function (14), the values of time constants $\tau_{j}$ and zero coefficient $\beta_{3}$ can be obtained as below

$$
\begin{aligned}
& \tau_{1}=B_{1}, \tau_{2}=\frac{B_{2}}{B_{1}}, \tau_{3}=\frac{B_{3}}{B_{2}}, \tau_{4}=\frac{B_{4}}{B_{3}}, \\
& \tau_{5}=\frac{B_{5}}{B_{4}}, \tau_{6}=\frac{B_{6}}{B_{5}}, \tau_{7}=\frac{B_{7}}{B_{6}}, \beta_{3}=\frac{A_{2}}{B_{2}}
\end{aligned}
$$

Thus, to realize the function in (7) the parameters in (15) can be determined by substituting the related coefficient values of (7) into (16), given by

$$
\begin{aligned}
& \tau_{7}=0.17463, \tau_{6}=0.36216, \tau_{5}=0.54492, \tau_{4}=0.78316, \\
& \tau_{3}=1.15600, \tau_{2}=1.87500, \tau_{1}=4, \beta_{3}=-0.29009
\end{aligned}
$$

One can denormalize (7) to any desired centre frequency according to application requirement. Herein, the centre frequency is selected around $100 \mathrm{MHz}$ as an example.

To improve transconductors matching and facilitate design automation, the transconductors in Fig. 1 are designed with identical transconductance $2.8 \mathrm{mS}$. Thus, the transconductance $\mathrm{g}_{\mathrm{a} 3}$ of the input transconductor can be calculated by (16) as $-0.812 \mathrm{mS}$, in which the negative transconductance can be realized simply by exchanging the two input or output terminals of the transconductor. It is worth noting that only one transconductor, $g_{a 3}$, is required to produce the transmission zeros in the realization of the desired bandpass Marr wavelet described in (7). This shows the simplicity of realization and is also desired for IC design due to the minimum number of components which implies low power consumption.

With parasitic capacitance $0.2 \mathrm{pF}$ taken into account, the capacitors values can be obtained by (17) as below

$C_{7}=1.0268 p \mathrm{~F}, C_{6}=2.0281 p \mathrm{~F}, C_{5}=3.0515 p \mathrm{~F}$,

$C_{4}=4.3857 p F, C_{3}=6.4736 p F, C_{2}=10.5000 p F$,

$C_{1}=22.4000 p F$

\section{Simulation RESUlts}

The wavelet filter is designed and simulated using standard TSMC $0.18 \mu \mathrm{m}$ CMOS process model with the following key model parameters, $\quad \mathrm{V}_{\mathrm{tn} 0}=0.37296 \mathrm{~V}, \quad \mathrm{~V}_{\mathrm{tp} 0}=-0.39018 \mathrm{~V}$, $\mathrm{K}_{\mathrm{n}}=173.9 \mu \mathrm{A} / \mathrm{V}^{2}$ and $\mathrm{K}_{\mathrm{p}}=-35.0 \mu \mathrm{A} / \mathrm{V}^{2}$. The total power consumption of the filter is only $24.87 \mathrm{~mW}$ with $1.8 \mathrm{~V}$ power supply. Fig. 3 plots the frequency response of the filter simulated by HSpice, achieving the peak value 1.83 at $\mathrm{f}=100$ $\mathrm{MHz}$. The $-3 \mathrm{~dB}$ frequencies of this filter are $71.9 \mathrm{MHz}$ and $123 \mathrm{MHz}$ respectively, which are almost the same as the ideal values. Simulation results have shown that the total harmonic distortion (THD) is below $1 \%$ for differential input signal swings up to $220 \mathrm{mVpp}$. The input equivalent noise is 26.2 $\mathrm{nV} / \sqrt{ } \mathrm{Hz}$ at $100 \mathrm{MHz}$, corresponding to a dynamic range of about $52 \mathrm{~dB}$. As seen from Fig. 3, the performance of the $\mathrm{Gm}-\mathrm{C}$ wavelet filter is confirmed by the excellent approximation of the Marr wavelet.

By changing the transconductance values $\mathrm{g}_{\mathrm{i}}$, or capacitance values $C_{i}$, the wavelet filters at different scales can be implemented accordingly. Fig. 4 depicts the frequency responses of the wavelet filter at dyadic scale values $\mathrm{a}=2^{1}$ and $\mathrm{a}=2^{-1}$, whose centre frequency is $50 \mathrm{MHz}$ and $200 \mathrm{MHz}$ respectively. Observed from these figures, simulation results indicate that the presented approach can easily implement the wavelet bases at different dilations and is very feasible for the application of WT in high and very high frequencies. 


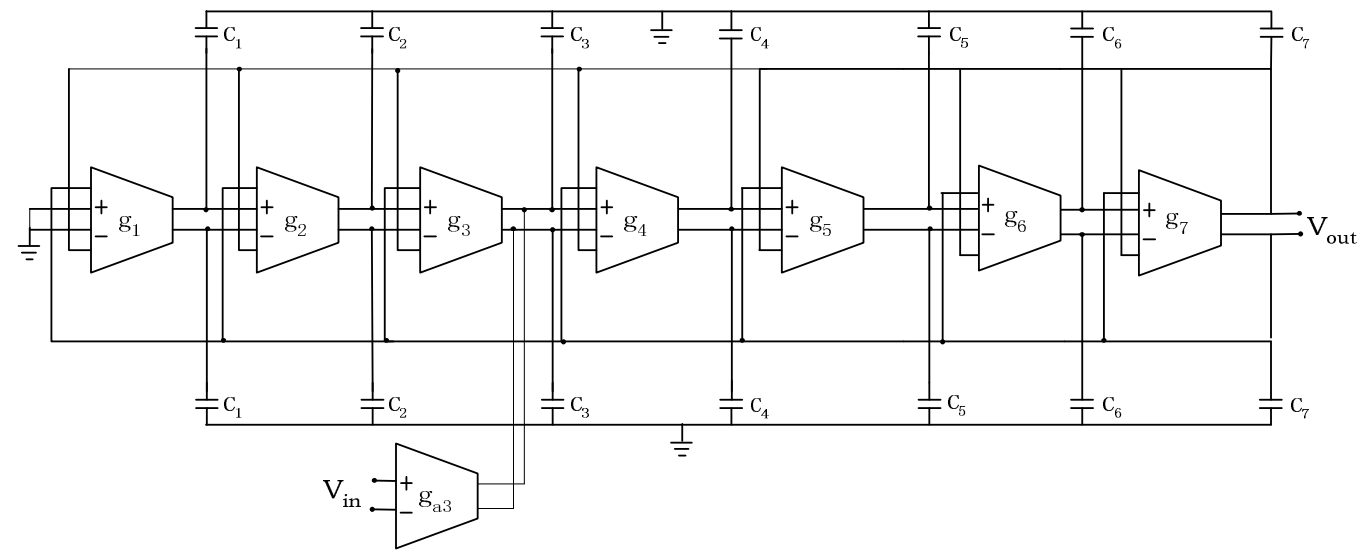

Figure 2. Seventh-order fully- differential IFLF bandpass filter with input distribution transconductor network

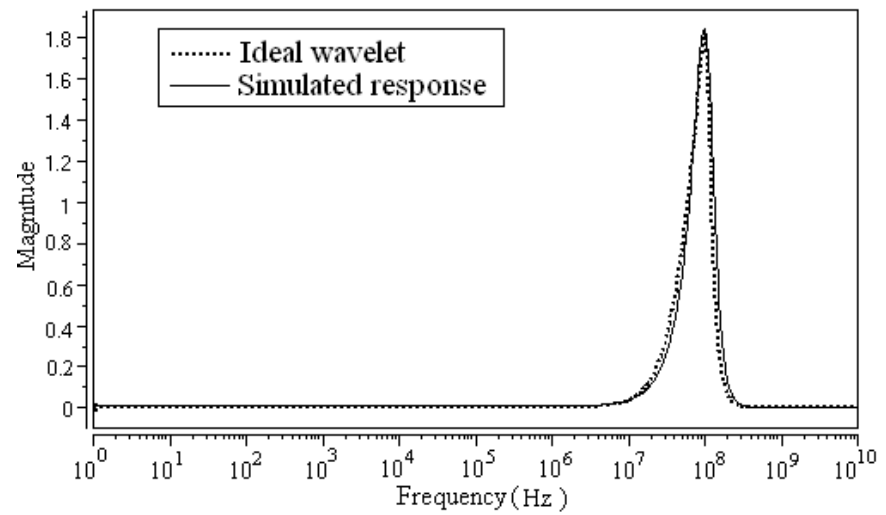

Figure 3. Frequency response of the wavelet filter at $a=1$

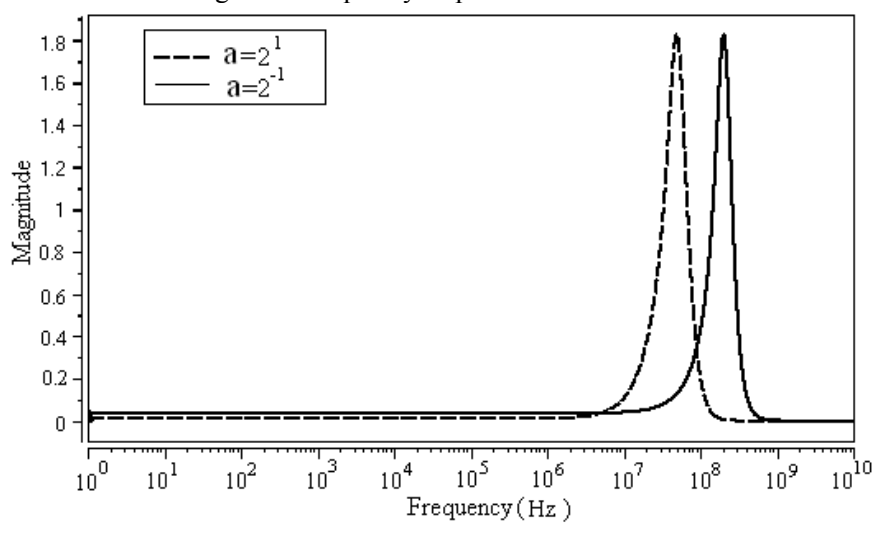

Figure 4. Frequency response of the wavelet filter at $a=2^{1}$ and $a=2^{-1}$

\section{CONCLUSIONS}

This paper has described a novel method for the analogue implementation of wavelet transform suitable for highfrequency real-time operation. Taking the Marr wavelet as an example, the proposed approach employs a fully-differential $\mathrm{Gm}-\mathrm{C}$ bandpass filter based on the IFLF MLF structure in the wavelet filter design, in which a full-differential linear transconductor is used as the $\mathrm{Gm}$ cell to enhance the performance. Simulation results using $1.8 \mathrm{~V} 0.18 \mu \mathrm{m}$ CMOS technology show an excellent approximation of the Marr wavelet base with only $24.87 \mathrm{~mW}$ power dissipation at $100 \mathrm{MHz}$ centre frequency. These results have shown that this wavelet filter can be used as the front-end stage of a real-time high-frequency signal analysis and recognition system.

\section{REFERENCES}

[1] S. Mallat, A Wavelet Tour of Signal Processing. New York: Academic Press, 2001.

[2] K. C. Ho, W. Prokopiw, and Y. T. Chan, "Modulation identification of digital signals by the wavelet transform," IEE Proc.- Radar, Sonar Navig., Vol. 147, No. 4, pp. 169176, 2000.

[3] M. Kawada, A. Tungkanawanich, Z. I. Kawasaki, and K. Matsu-ura, "Detection of wide-band E-M signals emitted from partial discharge occurring in GIS using wavelet transform," IEEE Trans. Power Delivery, Vol. 15, No. 2, pp. 467-471, 2000.

[4] D. E. Driscoll and S. D. Howard, "The detection of radar pulse sequences by means of a continuous wavelet transform,” Proc. IEEE ICASSP, pp.1389-1392, 1999.

[5] X. D. Ma, C. Zhou and I. J. Kemp, "DSP based partial discharge characterisation by wavelet analysis," Proc. ISDEIV, pp. 780-783, 2000.

[6] H. Liao, B. F. Cockburn and M. K. Mandal, "Efficient implementation of the discrete wavelet transform on the parallel DSP-RAM architecture," Proc. CCECE, pp. 11891192, 2001.

[7] Y. Sun, Design of High Frequency Integrated Analogue Filters. Stevenage: IEE Press, 2002.

[8] A. J. Casson, D. C. Yates, S. Patel, and E. RodriguezVillegas, "An analogue bandpass filter realization of the continuous wavelet transform," Proc. IEEE EMBS, pp. 1850-1854, 2007.

[9] C. S. Kim, Y. H. Kim, and S. B. Park, "New CMOS linear transconductor," Electron. Letters, Vol. 28, No. 21, pp. 1962-1964, 1992.

[10] Y. Sun and J. K. Fidler, "Synthesis and performance analysis of universal minimum component integrator-based IFLF OTA-grounded capacitor filter," IEE Proc. Circuits Devices Syst., Vol. 143, No. 2, pp. 107-114, April 1996.

[11] Y. Sun and J. K. Fidler, "OTA-C realization of general high-order transfer functions," Electron. Letters, Vol. 29, No. 12, pp.1057-1058, 1993. 